\title{
PZT Based Smart Aggregate for Unified Health Monitoring of RC Structures
}

\author{
Suraj N. Khante*, Shruti R. Gedam \\ Applied Mechanics Department, Government College of Engineering Amravati, Amravati, India \\ Email: *snkhante@yahoo.com, shruti.gedam27@gmail.com
}

Received 6 February 2016; accepted 26 February 2016; published 29 February 2016

Copyright (C) 2016 by authors and Scientific Research Publishing Inc.

This work is licensed under the Creative Commons Attribution International License (CC BY). http://creativecommons.org/licenses/by/4.0/

(c) 7 Open Access

\section{Abstract}

The most familiar civil engineering structure is reinforced concrete (RC) structure. Performance of structure undergoes changes during their service life with time. Thus, it is of great concern to monitor the health of RC structure. Structural health monitoring (SHM) is the art of detecting the changes in structure that influences its performance. Various techniques to monitor the health of structure are broadly studied worldwide. PZT based smart aggregate can play an effective role as an advanced tool in the development of structural health monitoring. This research work contributes for proposing a more generous Non-Destructive Evaluation (NDE) technique for structural health monitoring by using smart materials. If performance of a structure deviates from the design parameters with time, appropriate and effective maintenance is required. Considering the relevant need of RC structures, a more sensitive and cost-effective approach by using Electro-Mechanical Impedance (EMI) technique has been proposed for implementation in real-life situations. In general, surface bonded PZT transducer is used for SHM. Since PZT transducers are of very small dimension and brittle in nature, for consistent characteristics, they should be protected from severe environmental condition and other external interruptions. For this reason, PZT transducer is embedded in structure at the time of construction and manufacturing of the embedded transducer is simple. The proposed EMI technique assesses the health of RC structures more rationally by embedding PZT transducer in the structure, whose health is to be monitored over the user specified preset frequency range. The conductance and susceptance signatures are acquired by using LCR meter. At any future point of time, when it is desired to assess the health of structure, the conductance and susceptance signatures are acquired and further utilized for damage detection and quantification. The Root Mean Square Deviation (RMSD) is used to specify damage severity.

\section{Keywords}

Concrete, Impedance, Piezoelectric Materials, Sensors, Smart Aggregates

\footnotetext{
${ }^{*}$ Corresponding author.
} 


\section{Introduction}

Structures consist of an assembly of various members like columns, beams, slabs, etc. Piezoceramic transducer has been playing an important role in structural health monitoring. Any damage of the host structure reflects changes in its properties. Major damages to structures can lead to failures, causing inconvenience and safety problems. Therefore, continuous monitoring of structure is essential. Numerous structural engineers drew attention towards developing modern sensor technology for making structures, smart enough to warn the concerned authorities before their failure in the recent decade years.

Sensors like accelerometers, strain gauges, Piezo based Lead Zirconate Titanate (PZT) patches, etc. can be used to monitor the health of the structures. As compared to other sensors, piezo based sensors are becoming more popular because of their efficiency of monitoring. Smart materials are widely used in Structural Health Monitoring (SHM) systems due to the viability of them in host structures; to act as sensors and/or actuators to assess the continuous health of a structure.

Among all the health monitoring methods, Electro-Mechanical Impedance (EMI) based health monitoring using piezoelectric materials is extensively acknowledged. The conductance signature is acquired with the help of EMI active sensor which serves as an indication of damage in structure. Kaur, Gupta, Jain and Bhalla [1] presented the integrated application of the global vibration technique and the low-cost EMI technique for damage detection and severity assessment. Shanker [2] experimentally investigated the possibility of an embedded PZT as a sensor by using impedance based method over concrete beams and steel beams. Annamdas, Yang, and Soh [3] proposed a double protection wrap method employing both metal and non-metal of embedding PZT sensor in concrete for monitoring either fresh or cured concrete using embedded piezoelectric transducer (PZT) via EMI technique. Negi, Kaur, Bhalla and Chakraborty [4] presented the responses of PZT patches while embedded in host structure in three different configurations after embedding in a prototype RC beam. Dumoulin, Karaiskos and Deraemaeker [5] studied the embedded transducers to detect and follow the crack propagation in a reinforced concrete beam subjected to a three-point bending test. Song, $\mathrm{Gu}$ and Mo [6] proposed the multifunctionality of smart aggregates for early-age concrete strength monitoring, impact detection and structural health monitoring. Feng, Dandjekpo and Zhou [7] detected the post-earthquake damage in concrete dams by using embedded electro-mechanical impedance (EMI) sensors. Visalakshi and Bhalla [8] compared the sensing capabilities of surface bonded and embedded piezoceramic (PZT) patches in diagnosing chloride-induced corrosion for RC structures. Yoo, Khatibi and Kandare [9] reported a study on the degradation of embedded PZT transducers under cyclic loadings.

Damage identification is carried out by comparing the signature of structure in healthy state with the signatures obtained after a damage. The specific objective of the present study is to carry out the structural health monitoring of structures using indigenously prepared embedded PZT patches.

\section{Electro-Mechanical Impedance (EMI) Technique}

The Electro-Mechanical Impedance (EMI) technique using piezo elements is relatively a new technique for SHM. The EMI technique employs PZT patches as a sensor. A PZT patch is surface bonded or embedded inside the structures to be monitored, as shown in Figure 1. The sensing zone of PZT patch is limited to 0.4 to $2 \mathrm{~m}$.

The patch (length $2 l$, width $w$ and thickness $h$ ) behaves as thin bar undergoing axial vibration. When an alternating electric field is applied to the patch; it expands and contracts dynamically in direction " 1 ". Two end points of the patch can be assumed to encounter equal impedance $Z$ from the host structure. An electro-mechanical model of the system is shown in Figure 1(b), where the structure has been replaced by two equal mechanical impedances $Z$.

$$
Y=2 \omega j \frac{w l}{h}\left[\left(\varepsilon_{33}\right)^{\mathrm{T}}+\left(\frac{Z_{a}}{Z+Z_{a}}\right)\left(d_{31}\right)^{2} \bar{Y}^{E}\left(\frac{\tan k l}{k l}\right)-\left(d_{31}\right)^{2} \bar{Y}^{E}\right]
$$

Equation (1) represents the complex electro-mechanical admittance $Y$ of the coupled system.

Where, $Z_{a}$ is mechanical impedance of the PZT patch, $\omega$ is angular frequency and $k l$ is the wave number, $d_{31}$ is the piezoelectric strain coefficient of the PZT material, $\bar{Y}^{E}$ is the complex Young's modulus under constant electric field, $\left(\varepsilon_{33}\right)^{\mathrm{T}}$ is the complex electric permittivity at constant stress.

The mechanical impedance $Z$ of the host structure is the function of the structural parameters, i.e., as stiffness, damping and mass. Any damage to the structure will cause these parameters to change, and hence changes the 


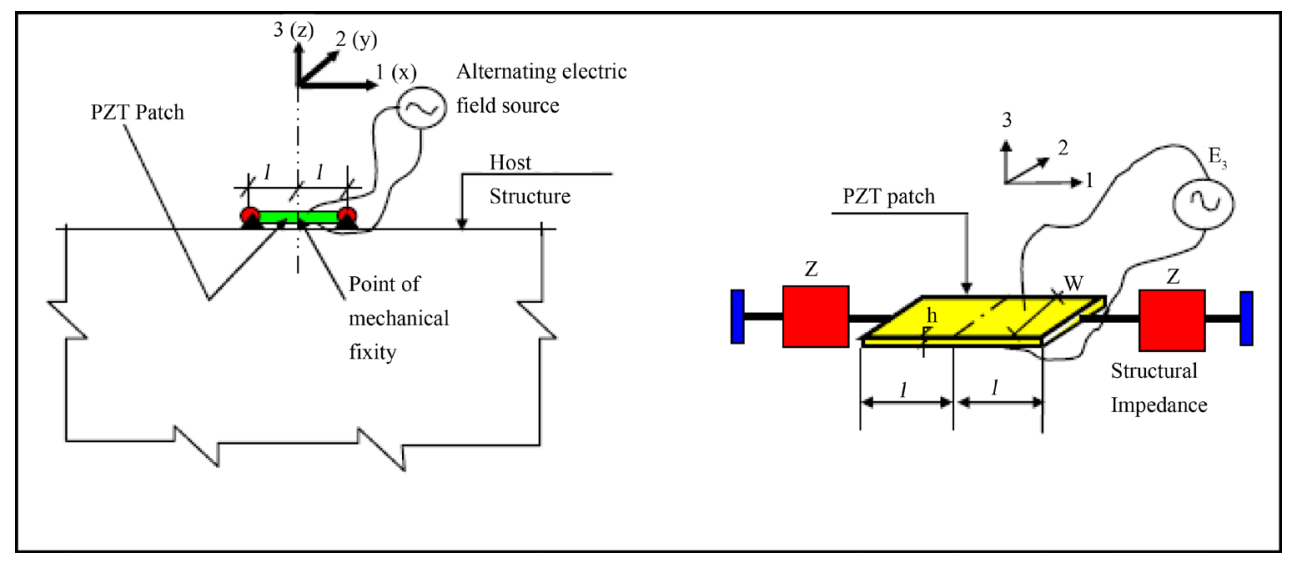

(a)

(b)

Figure 1. Modeling PZT-structure interaction [2]. (a) A PZT patch bonded to structure under electric excitation; (b) Interaction model of PZT patch and host structure.

drive point mechanical impedance $Z$. As a result, the electro-mechanical admittance $Y$ will change and this serves an indicator of the state of health of the structure. Equation (1) is utilized in EMI technique for damage detection. The electro-mechanical admittance $Y$ consists of the real and the imaginary component called conductance and susceptance respectively. Hence, the magnitude of complex admittance can be calculated as given in Equation (2):

$$
Y=\sqrt{G^{2}+B^{2}}
$$

where, $G$ = Conductance; real part of admittance,

$B$ = Susceptance; imaginary part of admittance.

There are several techniques available to quantify damages in structure. In the present work, to quantify damage in structure Root Mean Square Deviation (RMSD) technique is used which is given as:

$$
M=\sqrt{\frac{\sum\left(G_{2}-G_{1}\right)^{2}}{\sum G_{1}^{2}}} \times 100(\%)
$$

where, $M=$ Damage metric (Root Mean Square Deviation),

$G_{1}=$ Conductance before damage,

$G_{2}=$ Conductance after damage.

\section{Experimental Investigation}

\subsection{General}

In order to perform structural health monitoring of concrete structures using EMI technique, a model RC beam and smart aggregate was cast. The experimental investigation is carried for damage identification and crack detection. The instrument used is digital Impedance Analyzer (LCR meter E4980A) of frequency range of $20 \mathrm{~Hz}$ to $2 \mathrm{MHz}$ and its connecting fixture, and a USB cable and VEE Pro 9.32 for data acquisition. To monitor the health of a structure experiment was performed in two stages i.e. firstly for the healthy state of host structure and then for damaged state of structure.

\subsection{Materials and Specimen}

The test was carried out on reinforced concrete beam. The PZT sensor was fabricated and embedded inside the RC beam. To prepare the embedded sensor SMart AGgregate (SMAG), both the surfaces of naked PZT patch with the dimensions of $10 \mathrm{~mm} \times 10 \mathrm{~mm} \times 0.2 \mathrm{~mm}$ are welded to coaxial cable wires, respectively, and the bare PZT impedance sensors is formed. The form work of card board with $20 \mathrm{~mm}$ diameter was first filled with ce- 
ment mortar of proportion (1:2) to the half depth. After 7 days curing, the PZT $(5 \mathrm{H})$ patch was bonded on the top of the surface of the cement block using epoxy (Araldite). After 24 hours, the card board was filled to full depth with the cement mortar. After 7 days of curing, the card board was removed from cement block and was ready to use in any structure under construction as shown in Figure 2.

The performance of sensor was studied on the R.C. beam structure whose dimensions and properties are shown in Table 1. The typical block diagram of RC beam with SMart AGgregate (SMAG) is shown in Figure 3.

\subsection{Experimental Set-Up}

This experiment essentially involves a simply supported RC beam with embedded SMAG at $100 \mathrm{~mm}$ distance from right support and $30 \mathrm{~mm}$ distance from bottom of beam as shown in Figure 4. The instrument required to obtain signatures are digital Impedance Analyzer (LCR meter E4980A) and its connecting fixture and a USB cable and VEE Pro 9.32 for data acquisition. This test was performed in two stages i.e. in the first stage signatures for healthy condition of the beam and in second stage for the damaged condition of beam is obtained.

In the first stage, the soldered PZT patch in the form of SMAG was wired to Impedance Analyzer through Connecting Fixture of LCR meter. Then the frequency was swept through $100 \mathrm{kHz}$ to $400 \mathrm{kHz}$ i.e. the PZT patch transfers this vibrations to the structure through adhesive bond layer. These vibrations are transferred to structures and reflected back from the same PZT patch through waves, which will indicate the health of the structure. The required parameter i.e. Conductance $(G)$ is directly measured through LCR meter for all the values of frequency. From the obtained data, the graph of Conductance versus Frequency is plotted. These graphs are said to be the Conductance Signature or just a signature shown by particular sensor for specimen. This data is saved in excel format and can be available as and when required. The assembly for the experiment of EMI technique is as shown in Figure 5.

In the second stage, damage was introduced in the form of significant visible crack by loading the specimen under Universal Testing Machine (UTM) as shown in Figure 6 and then the same damaged specimen with PZT patch was reanalyzed for damaged state by using the same procedure as previously adopted. The PZT patch again acts as an actuator and sensor. Then the data for healthy state and damaged state was compared.

Table 1. Material properties and dimensions of RC beam.

\begin{tabular}{cc}
\hline Dimensions/Properties & Value \\
Length (L) & $410 \mathrm{~mm}$ \\
Cross-section & $100 \mathrm{~mm} \times 100 \mathrm{~mm}$ \\
Grade of cement (OPC) & 43 \\
Grade of steel & Fe 415 \\
Reinforcement bars & 2\#8 $\mathrm{mm}$ dia at top and bottom \\
stirrups & $6 \mathrm{~mm}$ dia @80 mm c/c \\
Characteristic strength of concrete & $20 \mathrm{~N} / \mathrm{mm}^{2}$ \\
Curing period & 7 days \\
\hline
\end{tabular}

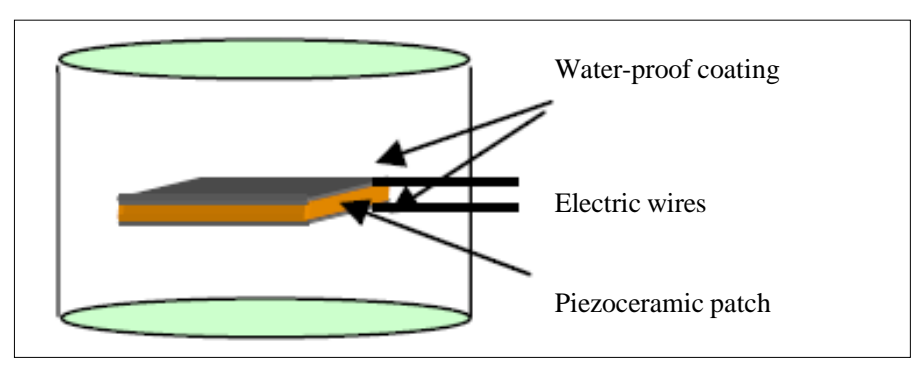

Figure 2. Typical SMart AGgregate (SMAG). 


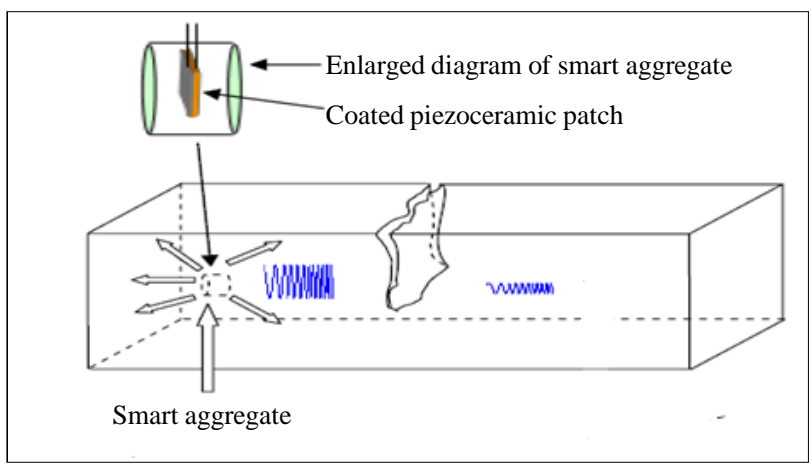

Figure 3. Block diagram of RC beam with embedded SMAG.

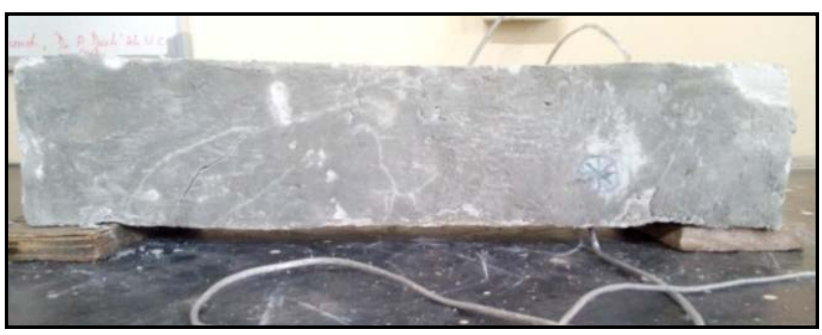

Figure 4. RC beam with embedded SMAG.

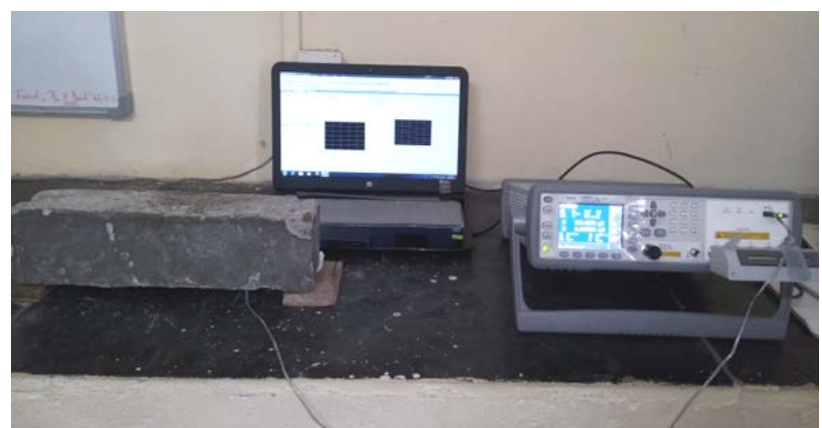

Figure 5. General configuration of set-up and instrument using E4980A impedance analyzer.

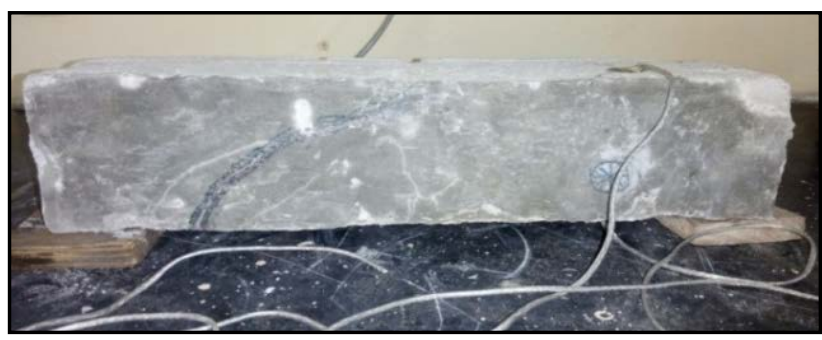

Figure 6. Damaged RC beam.

\section{Results and Discussions}

\subsection{Response for Healthy Condition of Embedded SMAG}

For the first stage, the healthy structure response is taken, with the SMAG embedded in simply supported RC beam. Piezoelectric patch was connected via cable to LCR meter and corresponding response was recorded. The beam was subjected to frequency range of 100 to $400 \mathrm{kHz}$. In this range, corresponding conductance values 
were recorded by LCR meter. The conductance graph is shown in Figure 7 and susceptance graph is shown in Figure 8. The conductance graph shows abundant peaks where the susceptance graph is comparatively flat.

\subsection{Response for Damaged Condition}

In the second stage, the beam is laterally loaded on Universal Testing Machine until significant visible crack is seen. The same beam with embedded SMAG was connected via cable to LCR meter and corresponding response in term of conductance and susceptance was recorded as shown in Figure 9 and Figure 10 respectively.

By comparing the results obtained for both the stages as shown in Figure 11 and Figure 12, it can be said that the responses for RC beam is different for healthy and damaged conditions. Changes in the responses are the indications of damages. The effectiveness of embedded PZT is evident from the fact that there is clear shift in vertical as well as lateral direction in the recorded conductance response. The absence of peaks in the original susceptance response in Figure 8 and Figure 10 and lesser deviation from original curve in Figure 12 makes susceptance signature a weak candidate for flaw detection. Structural health monitoring with embedded piezoelectric patches proves to be effective tool for damage detection.

The damage index recorded by embedded SMAG using root mean square deviation technique (RMSD) is found to be $5.0014 \%$ in frequency range of $100-400 \mathrm{kHz}$.

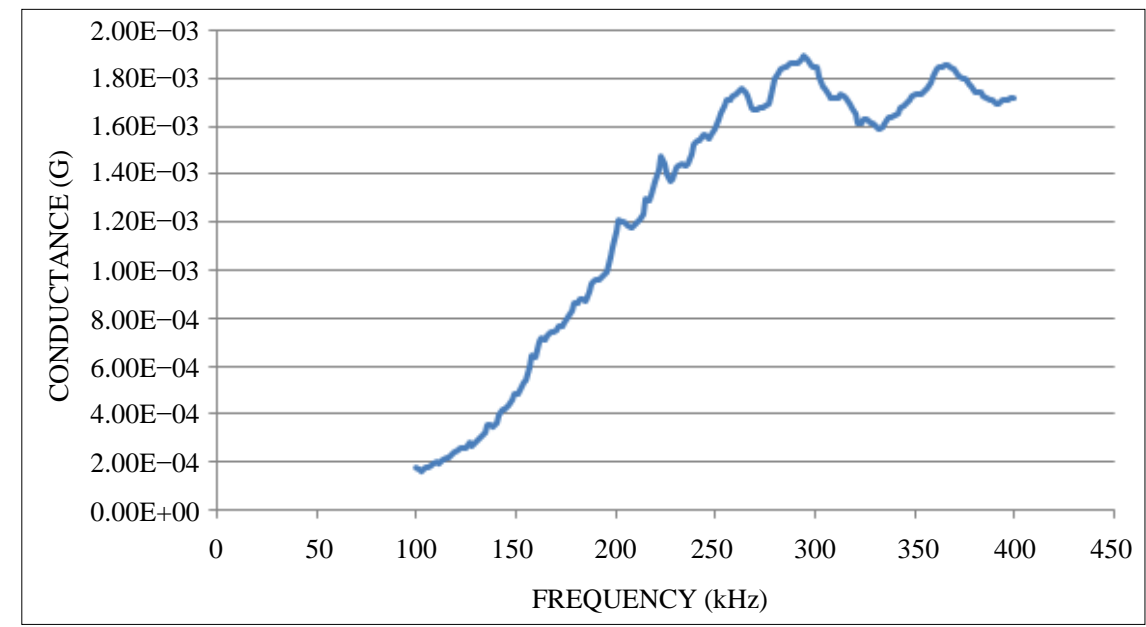

Figure 7. Conductance graph for healthy beam by embedded SMAG.

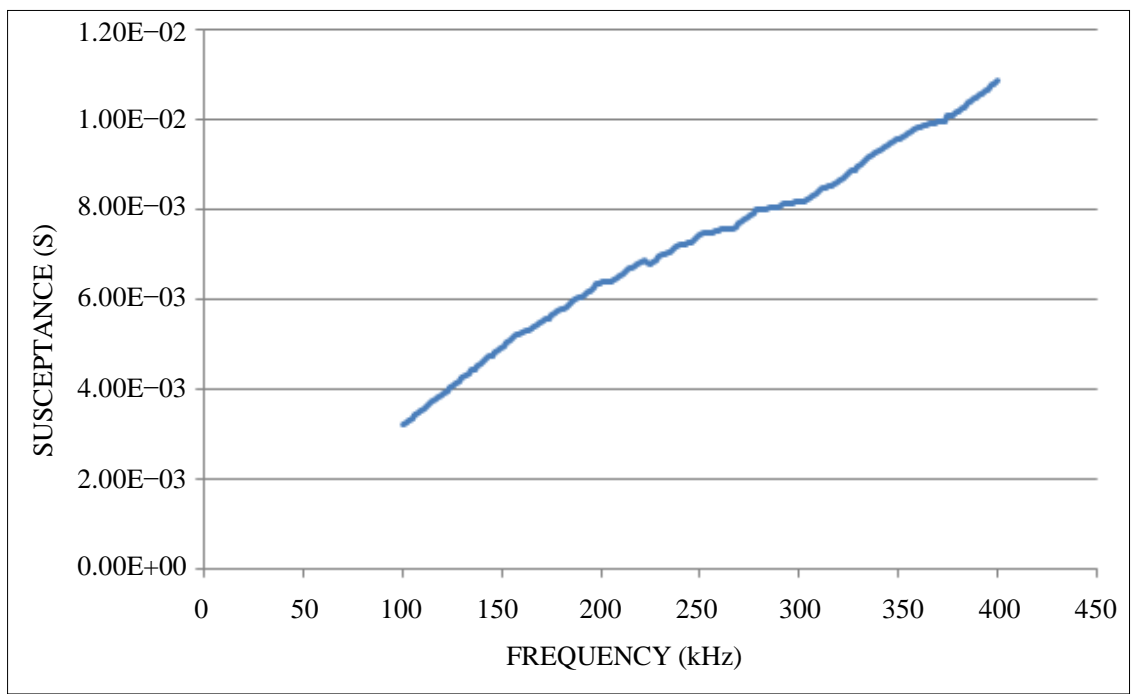

Figure 8. Susceptance graph for healthy beam by embedded SMAG. 


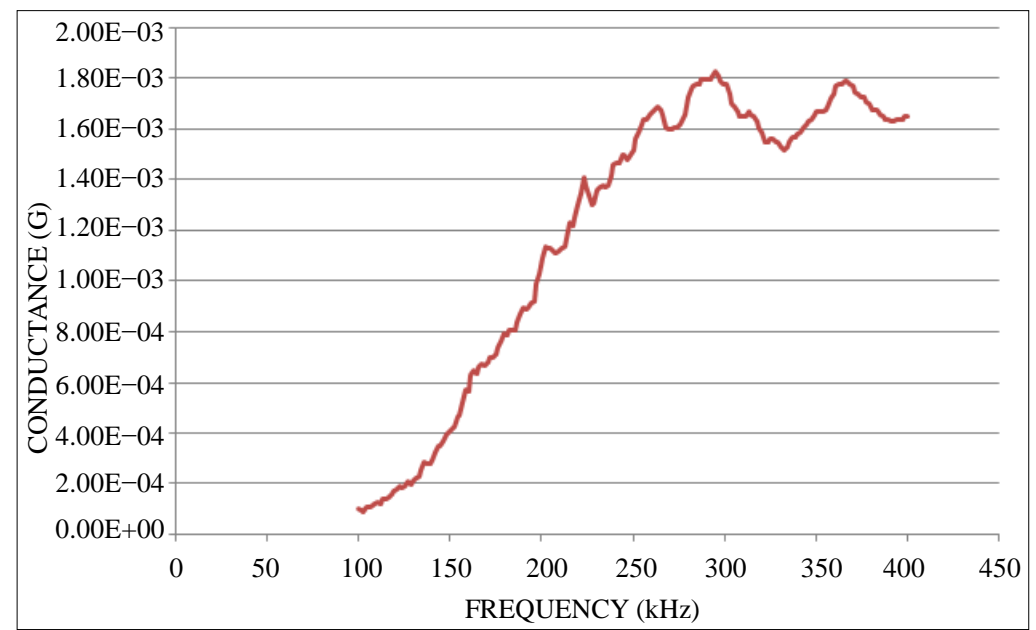

Figure 9. Conductance graph for damaged beam by embedded SMAG.

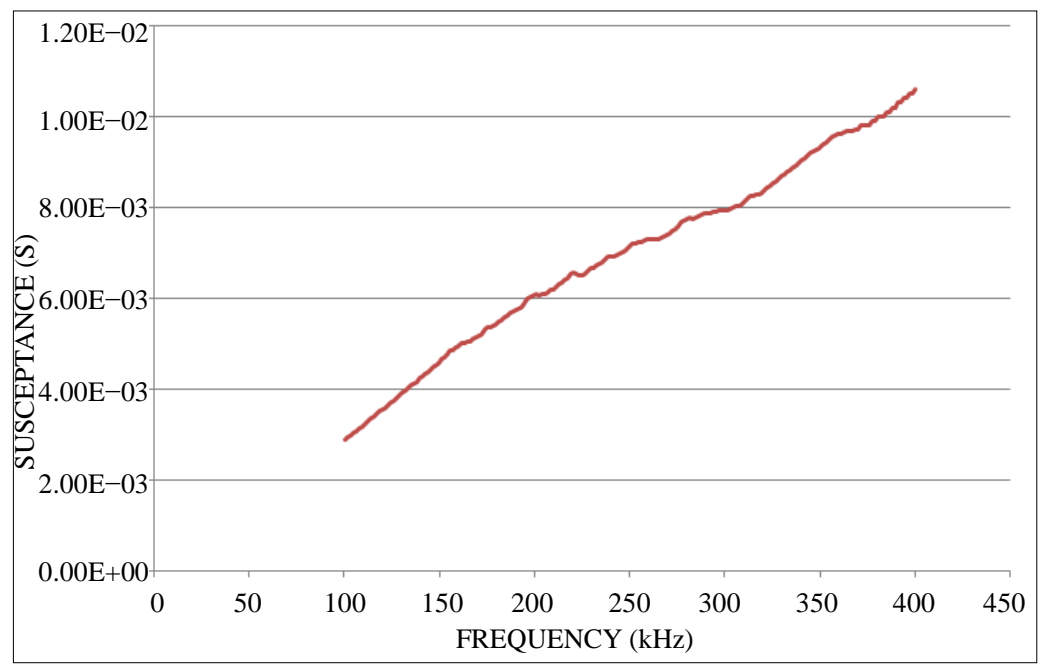

Figure 10. Susceptance graph for damaged beam by embedded SMAG.

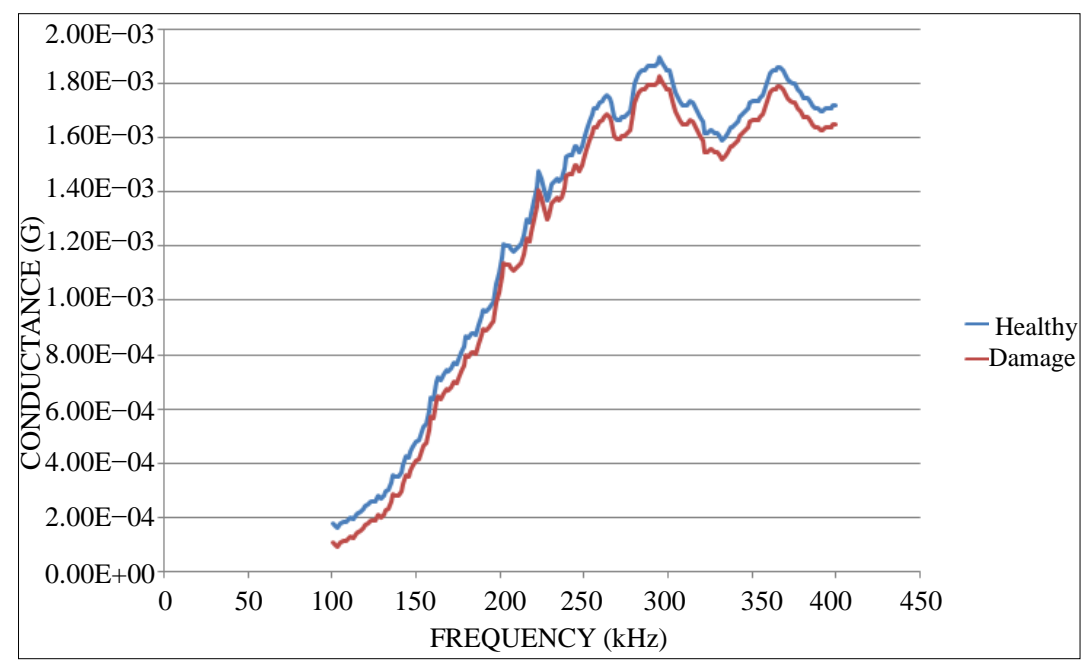

Figure 11. Conductance graph for healthy and damaged beam by embedded SMAG. 


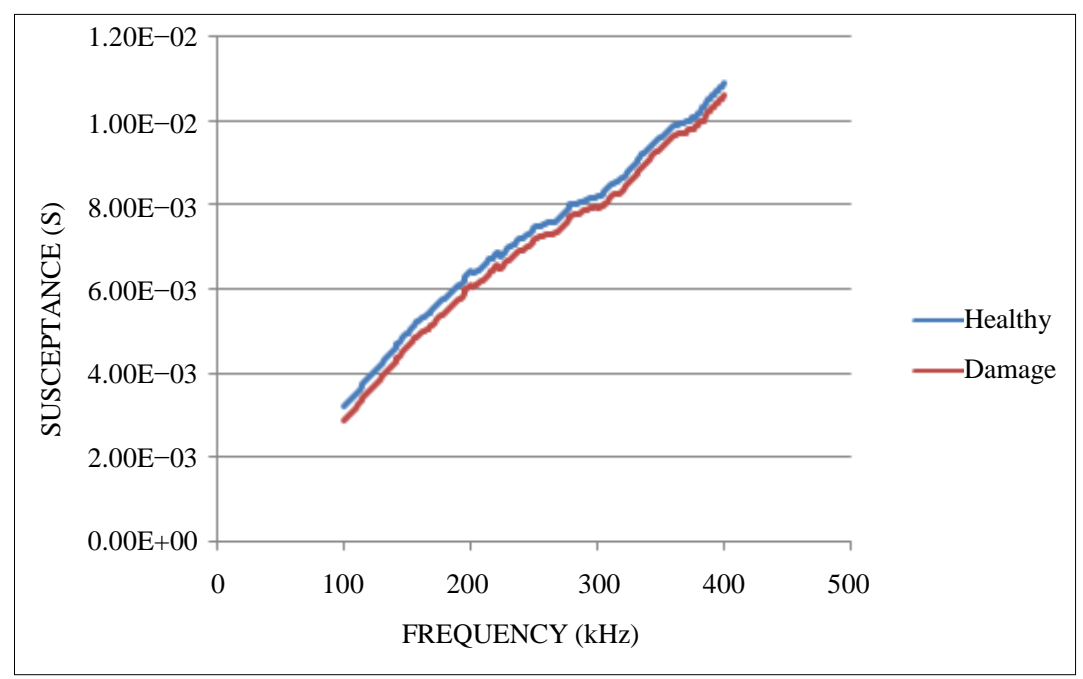

Figure 12. Susceptance graph for healthy and damaged beam by embedded SMAG.

\section{Conclusion}

It is concluded from the above experimental work that continuous health monitoring of structure is possible using proposed simple low cost technique. Instead of surface bonded PZT patches, structural health monitoring with embedded PZT patches is very effective and easy to implement and the record data can be available as and when required. For health monitoring of RC structure, it is a challenging task to detect incipient damages. It is found that the incipient level damages are very quickly and effectively detected by using present EMI technique. It is again established that the conductance signature is more effective in damage detection than susceptance signature.

\section{References}

[1] Kaur, N., Gupta, N., Jain, N. and Bhalla, S. (2013) Integrated Global Vibration and Low-Cost EMI Technique for Structural Health Monitoring of RC Structures Using Embedded PZT Patches. Proceedings of UKIERI Congress on Innovations in Concrete Construction, B. R. Ambdekar National Institute of Technology, Jalandhar, 5-8 March 2013, Paper No. UCC-488.

[2] Shanker, R. (2009) An Integrated Approach for Structural Health Monitoring. Indian Institute of Technology, Delhi.

[3] Annamdas, V., Yang, Y. and Soh, C. (2010) Impedance Based Concrete Monitoring Using Embedded PZT Sensors. International Journal of Civil and Structural Engineering, 1, 414:424.

[4] Negi, P., Kaur, N., Bhalla, S. and Chakraborty, T. (2014) Experimental Strain Sensitivity Investigations on Embedded PZT Patches in Varying Orientations. Proceedings of $9^{\text {th }}$ Biennial Conference on Structural Engineering Convention (SEC 2014), New Delhi, 22-24 December 2014, 2615-2620.

[5] Dumoulin, C., Karaiskos, G. and Deraemaeker, A. (2015) Monitoring of Crack Propagation in Reinforced Concrete Beams Using Embedded Piezoelectric Transducers. In: Ohtsu, M., Ed., Acoustic Emission and Related Non-Destructive Evaluation Techniques in the Fracture Mechanics of Concrete, Woodhead Publishing, Cambridge. http://dx.doi.org/10.1016/b978-1-78242-327-0.00008-8

[6] Song, G.B., Gu, H.C. and Mo, Y.-L. (2008) Smart Aggregates: Multi-Functional Sensors for Concrete Structures-A Tutorial and a Review. Smart Materials and Structures, 17, Article ID: 033001, 17 p.

[7] Feng, X., Dandjekpo, E.T. and Zhou, J. (2012) Post-Earthquake Damage Detection Using Embedded Electro-Mechanical Impedance Sensors for Concrete Dams. http://www.iitk.ac.in/nicee/wcee/article/WCEE2012_0258.pdf

[8] Visalakshi, T. and Bhalla, S. (2014) Reinforcement Corrosion Assessment Capability of Surface Bonded and Embedded Piezo Sensors for RC Structures. Journal of Intelligent Material Systems and Structures, 1-10.

[9] Yoo, S., Khatibi, A.A. and Kandare, E. (2014) Durability of Embedded PZTs in Structural Health Monitoring Systems under Cyclic Loading. Advanced Materials Research, 891-892, 1255-1260. http://dx.doi.org/10.4028/www.scientific.net/AMR.891-892.1255 